\title{
Frontotemporal Connections in Episodic Memory and Aging: A Diffusion MRI Tractography Study
}

\author{
Claudia Metzler-Baddeley, ${ }^{1}$ Derek K. Jones, ${ }^{1}$ Boubakeur Belaroussi, ${ }^{2}$ John P. Aggleton, ${ }^{1}$ and Michael J. 0'Sullivan ${ }^{1}$ \\ ${ }^{1}$ Cardiff University Brain Research Imaging Centre, School of Psychology, and Neuroscience and Mental Health Research Institute, Cardiff University, \\ Cardiff CF10 3AT, United Kingdom, and ²BioClinica SAS, F-69008 Lyon, France
}

Human episodic memory is supported by networks of white matter tracts that connect frontal, temporal, and parietal regions. Degradation of white matter microstructure is increasingly recognized as a general mechanism of cognitive deterioration with aging. However, atrophy of gray matter regions also occurs and, to date, the potential role of specific white matter connections has been largely ignored. Changes to frontotemporal tracts may be important for the decline of episodic memory; while frontotemporal cooperation is known to be critical, the precise pathways of interaction are unknown. Diffusion-weighted MRI tractography was used to reconstruct three candidate fasciculi known to link components of memory networks: the fornix, the parahippocampal cingulum, and the uncinate fasciculus. Age-related changes in the microstructure of these tracts were investigated in 40 healthy older adults between the ages of 53 and 93 years. The relationships between aging, microstructure, and episodic memory were assessed for each individual tract. Age-related reductions of mean fractional anisotropy and/or increased mean diffusivity were found in all three tracts. However, age-related decline in recall was specifically associated with degradation of fornix microstructure, consistent with the view that this tract is important for episodic memory. In contrast, a decline in uncinate fasciculus microstructure was linked to impaired error monitoring in a visual object-location association task, echoing the effects of uncinate transection in monkeys. These results suggest that degradation of microstructure in the fornix and the uncinate fasciculus make critical but differential contributions to the mechanisms underlying age-related cognitive decline and subserve distinct components of memory.

\section{Introduction}

Understanding mechanisms of cognitive aging is an important challenge given the changing demographics of Western society. Although episodic memory decline is a core component, and much is known about putative networks for episodic memory, age-related variation has rarely been related to structural change within these specific networks. Episodic memory is supported by networks spanning frontal, temporal, and parietal lobes (Fletcher and Henson, 2001; Simons and Spiers, 2003; Cabeza et al., 2008). The various interconnections are presumed to enable distinct aspects of memory (Aggleton and Brown, 2006; Wilding and Herron, 2006; Daselaar et al., 2009). Whereas the differential effects of gray matter lesions have been extensively studied (Ranganath, 2010), less is known about the interconnecting fiber tracts. In aging, degradation of white matter structure has emerged as an important general factor (Barrick et al., 2010; O'Sullivan et al., 2001), further focusing attention on the critical

\footnotetext{
Received May 10, 2011; revised June 29, 2011; accepted July 26, 2011.

Author contributions: C.M.-B., D.K.J., J.P.A., and M.J.O. designed research; C.M.-B. performed research; C.M.-B. and B.B. analyzed data; C.M.-B., D.K.J., J.P.A., and M.J.O. wrote the paper.

This work was supported by the Medical Research Council, UK, via a Clinician Scientist Fellowship to M.J.0. (G0701912). We thank Ofer Pasternak for his assistance with the partial volume corrections of the diffusion MRI data. The authors declare no financial conflict of interest.

Correspondence should be addressed to Claudia Metzler-Baddeley, School of Psychology, Cardiff University, Tower Building, Park Place, CardiffCF10 3AT, UK. E-mail: Metzler-BaddeleyC@cardiff.ac.uk.

DOI:10.1523/JNEUROSCI.2317-11.2011

Copyright $\odot 2011$ the authors $\quad 0270-6474 / 11 / 3113236-10 \$ 15.00 / 0$
}

white matter connections. In monkeys, selective tract transections, e.g., of the uncinate fascicularis or fornix (Gaffan and Eacott, 1995a,b; Gaffan and Wilson, 2008), only partially reproduce the memory deficits that follow disconnection of prefrontal and temporal lobes. Further, homology with human white matter anatomy is uncertain and lesion studies cannot model varying degrees of simultaneous damage to multiple tracts, as may occur in aging. Diffusion-weighted magnetic resonance imaging (DWI) (Jones et al., 1999a; Basser et al., 2000) provides a means to surmount these problems through in vivo reconstruction, visualization, and quantification of white matter microstructure.

The present study examined three tracts that link frontotemporal regions (Schmahmann and Pandya, 2006) and are implicated in episodic memory by either experimental transections or rare isolated lesions in humans (Valenstein et al., 1987; Levine et al., 1998; Aggleton et al., 2000). The fornix connects the hippocampal formation to the prefrontal cortex (Poletti and Creswell, 1977). The uncinate fasciculus (UF) is a bidirectional pathway that links the anterior temporal lobe with the orbital and medial prefrontal cortex (Crosby et al., 1962; Schmahmann and Pandya, 2006), providing an afferent sensory route for prefrontal cognitive functions (Gaffan and Wilson, 2008). The parahippocampal cingulum (PHC) (Mufson and Pandya, 1984) includes fibers that link the hippocampal formation with the cingulate cortex (Vann et al., 2009), thereby providing a second route for frontotemporal interactions.

Recent DWI studies have found that variations in fornix microstructure in young people correlate selectively with recollec- 
tive memory (Rudebeck et al., 2009), closely supporting clinical studies of fornix pathology (Aggleton et al., 2000; Tsivilis et al., 2008). As only the fornix was examined, the specificity of this association remains unclear. Variations in UF microstructure have also been linked to aspects of memory in older adults (Sasson et al., 2010) and children (Mabbott et al., 2009). Our study investigated the specific relationships between microstructure of all three tracts and age-related decline of episodic memory. This is also the first diffusion study to resolve an important confound in aging studies-the effect of atrophy on measures of microstructure (Concha et al., 2005) - through rigorous correction for partial volume artifacts (Pasternak et al., 2009).

\section{Materials and Methods}

Participants. Participants were recruited from the local community by advertisements posted in family physician waiting rooms, newsletters, bulk mail advertisements, and via the School of Psychology Community Panel of healthy research volunteers at Cardiff University. Forty-six individuals (21 males and 25 females) between the ages of 53 and 93 years were recruited. Exclusion criteria were as follows: a history of neurological disease or mental disorder (clinical disorders or acute medical conditions/physical disorders in the Diagnostic and Statistical Manual of Mental Disorders), including past history of moderate to severe head injury, prior or current alcohol and/or drug abuse, symptomatic memory or other cognitive function decline, and previous stroke or cerebral hemorrhage. Significant vascular disease elsewhere (peripheral vascular disease, carotid or vertebral artery stenosis, or previous coronary intervention), structural heart disease or heart failure, and contra-indications to MRI were also exclusion criteria. One participant did not complete the study due to ill health and another withdrew. One participant was excluded because of subsequent diagnosis of Parkinson's disease. Structural MRI scans (fluid attenuating inversion recovery and T1weighted) were inspected for overt pathology; three participants were excluded because of extensive white matter hyperintensities suggestive of significant cerebral microvascular disease (Fazekas et al., 1993) and one participant was excluded due to severe motion artifact. The oldest participant was partially sighted and was, therefore, not assessed in any tasks requiring vision. Thus, 39 MRI and 40 cognitive datasets were available for the final analyses, although one of the cognitive datasets lacked visual task information. Please see Table 1 for a summary of participants' background demographic information and cognitive test results.

Cognitive testing. Detailed assessment of memory and executive functions was performed over two $1.5 \mathrm{~h}$ testing sessions (Table 1).

Verbal intelligence was assessed with the National Adult Reading TestRevised (Nelson, 1991) and the Graded Naming Test (McKenna and Warrington, 1980) with semantic memory assessment provided by the Pyramids and Palm Tree Test (Howard and Patterson, 1992). Verbal working memory span was assessed with the Digit Span forwards task from the Wechsler Adult Intelligence Scale- third UK Edition (WAIS-III UK) (Wechsler, 1999) and visual memory span with the Visual Pattern Test (Della Sala et al., 1997). Different aspects of executive functions were measured: reversal of digit sequences was assessed with the Digit Span backwards task from the WAIS-III and attention switching was examined with a version of the verbal trails test that required alternation between letters and digits (Baddeley, 1996). The suppression of responseincongruent information was measured with the Stroop test (Trenerry et al., 1989), and problem solving with the Tower of London test from the Delis and Kaplan Executive Function System battery (D-KEFS) (Delis et al., 2001). Verbal generation and fluency were measured with the verbal fluency tests from the D-KEFS for letters F, A, and S and for the categories of animals and boys' names. In addition, the Digits Symbol Substitution test from the WAIS-III provided measures of response speed and focused attention. All participants were also screened for depression with the 15-item Geriatric Depression Scale (Sheikh and Yesavage, 1986). Participants in the present study were generally high functioning and performed within the normal to superior range in the executive tests across all ages (Table 1).
Table 1. Participants' demographics and neuropsychological assessment scores

\begin{tabular}{ll}
\hline Demographics & \\
\hline$n$ & 40 \\
Age & $\mathrm{M}=67.9, \mathrm{SD}=8.6(53-93$ years $)$ \\
Sex & 23 female, 17 male \\
Handedness & 38 right, 2 left \\
Education & $\mathrm{M}=15.3, \mathrm{SD}=3.03$ (10-22 years) \\
IQ and executive functions, mean (SD) & \\
National Adult Reading Test-IQ & $121(8)$ \\
Graded naming test & $25(3)(\max , 25)$ \\
Pyramids and Palm Trees & $51.5(0.7)(\max , 52)$ \\
Digit span forward/backward & $10.3(1.9)(\max , 16) / 8.2(2.4)(\max , 14)$ \\
Visual Span & $6.9(1.8)(\max , 15)$ \\
Verbal Trails (switching cost/errors) & $46(29) / 1.3(1.7)$ \\
Letter/Category Fluency & $47(13.5) / 42(10)$ \\
Stroop suppression score & $94.4(18)(\max , 112)$ \\
Tower of London achievement score & $17.5(4.4)(\max , 30)$ \\
Digit Symbol & $59.7(17.3)(\max , 133)$ \\
Geriatric Depression Scale-15 & $1.2(1.5)(\max , 15)$ \\
Episodic memory, mean (SD) & \\
Doors and People & \\
Immediate verbal recall & \\
$\quad$ Delayed verbal recall & $26.8(6.3)(\max , 36)$ \\
Immediate visual recall & $10.4(2.2)(\max , 12)$ \\
Delayed visual recall & $32.5(4.4)(\max , 36)$ \\
Verbal recognition & $11.6(0.9)(\max , 12)$ \\
Visual recognition & $16.9(3.3)(\max , 24)$ \\
Free and Cued Selective Reminding Test & $19.4(3.0)(\max , 24)$ \\
Immediate recall & \\
Free recall & $15.5(0.8)(\max , 16)$ \\
Total recall & $33.1(8.2)(\max , 48)$ \\
Delayed free recall & $46.5(3)(\max , 48)$ \\
$\quad$ Delayed total recall & $12.3(3.30)(\max , 16)$ \\
Paired association learning & $15.5(1.2)(\max , 16)$ \\
$\quad$ Level achieved & \\
Error ratio & $4.1(1.4)(\max , 8)$ \\
\hline & $0.1(0.03)$ \\
\hline
\end{tabular}

Episodic memory was assessed with instruments selected to tap into different aspects of memory processing. Both free recall (recollection) and recognition memory were assessed. The recognition tests should provide a partial measure of familiarity-based processes (Yonelinas, 2002). Memory performance was assessed in the visual and the verbal domain, both immediately after learning and after a delay. In addition, a measure of associative memory for object-location pairings, thought to be sensitive to medial temporal lobe (MTL) function (Blackwell et al., 2004), was included. Recall and recognition that were matched for difficulty were provided by the Doors and People test (Baddeley et al., 1994). This test provides raw scores for immediate and delayed visual and verbal recall, visual recognition, and verbal recognition. The Free and Cued Selective Reminding Test (FCSRT) (Grober and Buschke, 1987) was chosen to provide the following measures of verbal learning and memory: verbal encoding (immediate recall), free recall after a brief distraction period, total recall (free and cued recall for words that have not been free recalled) after brief distraction, delayed free recall, and delayed total recall (free and cued recall). Finally, associative visual object-location memory was assessed with a paper and pencil paired-associate learning (PAL) task requiring the participant to remember the locations of up to eight visual symbols. Dependent measures from the PAL were the number of symbol-location pairs that could be reliably recalled (level) and the error ratio (i.e., the number of errors proportional to the total number of locations). Table 1 summarizes the mean scores in the different episodic memory tests.

Diffusion MRI scanning. Diffusion-weighted MR data were acquired using a 3T GE HDx MRI system (General Electric Healthcare) with a twice-refocused spin-echo echo-planar imaging sequence providing whole oblique axial (parallel to the commissural plane) brain coverage. Data acquisition was peripherally gated to the cardiac cycle. Data were 
acquired from 60 slices of $2.4 \mathrm{~mm}$ thickness, with a field of view of $23 \mathrm{~cm}$, and an acquisition matrix of $96 \times 96$. TE was $87 \mathrm{~ms}$ and parallel imaging (ASSET factor $=2$ ) was used. The b-value was $1000 \mathrm{~s} / \mathrm{mm}^{2}$. In each imaging session, data were acquired with diffusion encoded along 30 isotropically distributed directions and three non-diffusion-weighted scans according to an optimized gradient vector scheme (Jones et al., 1999b). The acquisition time was $\sim 13 \mathrm{~min}$. The acquired images were corrected for distortions introduced by the diffusion-weighting gradients and for between-slice motion with appropriate reorienting of the encoding vectors (Leemans and Jones, 2009) before a model was fitted to the data to estimate the diffusion orientation in each voxel (Basser et al., 1994; Tournier et al., 2004).

Tractography and tract-specific measures. Tractography (Basser et al., 2000; Catani et al., 2002) based on the diffusion tensor model has been shown to generate anatomically plausible and reproducible reconstructions of tracts within regions of coherently oriented fibers (Catani et al., 2002; Malykhin et al., 2008). However, fiber tractography based on the tensor model is less successful in reconstructing the fibers of the fornix because of its close proximity to other white matter tracts (e.g., anterior commissure). In such settings, a technique that can resolve separate peaks in fiber orientation due to crossing fiber populations can maintain the faithfulness of the tracking algorithm to the tract in question and avoid incorrect, aberrant results (Pierpaoli et al., 2001). Deterministic tracking based on constrained spherical deconvolution (Tournier et al., 2004, 2007, 2008), which extracts peaks in the fiber orientational density function in each voxel, was therefore chosen as a more appropriate technique for fornix tractography. The fornix is also particularly susceptible to CSF contamination and hence partial volume artifacts (Concha et al., 2005; Jones and Cercignani, 2010), which may lead to an underestimation of anisotropy and premature termination of deterministic fiber tracking. Given that aging is associated with tissue atrophy (Ge et al., 2002; Smith et al., 2007), this was a critical methodological issue.

To ameliorate the effects of partial volume, the multiple tensor variance approach was used to model the data and correct fractional anisotropy (FA) and mean diffusivity (MD) measures on a voxel-by-voxel basis (Pierpaoli and Jones, 2004; Pasternak et al., 2009). Comparisons with uncorrected FA and MD values suggested that this approach effectively eliminated the effect of these errors on age correlations.

Tractography was performed using ExploreDTI (Leemans et al., 2009). The deterministic tracking algorithm estimated the principal diffusion orientation at each seed point and propagated in $0.5 \mathrm{~mm}$ steps along this direction. The fiber orientation(s) was/were then estimated at the new location and the tracking moved a further $0.5 \mathrm{~mm}$ along the direction that subtended the smallest angle to the current trajectory. In this way, a pathway was traced through the data until either FA fell below an arbitrary threshold (in this case 0.15 ) or the direction of the pathway changed through an angle $>60^{\circ}$.

Whole-brain tractography was performed using every voxel as a seed point. Three-dimensional reconstructions of the three tracts (Fig. 1) were then extracted from whole-brain tractograms by applying multiple waypoint regions of interest (ROIs) masks. ROIs defined tracts based on
Booleian logical operations; for example, selecting fibers that traversed ROI-1 AND ROI-2 but NOT ROI-3. Representative ROIs for the three tracts are shown in Figure 2. All ROIs were manually drawn in native space on color-coded fiber orientation maps (Pajevic and Pierpaoli, 1999) for each individual dataset by a single operator blinded to the participants' ages and cognitive scores, using landmark techniques that have previously been shown to be highly reproducible (Catani et al., 2002). These are detailed for each tract below. The mean FA and MD were then calculated for all reconstructed pathways in ExploreDTI by averaging the values sampled at each $0.5 \mathrm{~mm}$ step along the pathways (Jones et al., 2005), providing tract-specific means of FA and MD for the left and the right UF, left and right PHC, and the fornix. The precise neuropathological correlates of FA and MD are not known but these measures were adopted as they are the most widely used, show agerelated change (O'Sullivan et al., 2001), and FA has been shown to have neurophysiological relevance (Stufflebeam et al., 2008).

Fornix. A seed point ROI (Fig. $2 A$, blue) was placed medially on a coronal slice around the fornix bundle at the level of the entry point of the anterior pillars into the body of the fornix, approximately below the 
sagittal midline of the corpus callosum. An AND ROI (Fig. 2 A, green) was defined on an axial slice capturing the crus fornici in both hemispheres at the level of the inferior border of the splenium of the corpus callosum. NOT ROIs (Fig. $2 \mathrm{~A}$, red) were drawn on coronal slices rostral to the anterior fornix pillars and caudal to the crus fornici as well as on axial slices through the corpus callosum and the upper pons to exclude streamlines from the corpus callosum and the corticospinal tract. After visual inspection, obvious anatomically implausible outlier streams, if present, were removed using additional NOT ROIs.

Uncinate fasciculus. A seed point ROI was drawn on a coronal slice around the region where the UF enters the frontal lobe immediately rostral to the genu of the corpus callosum (Fig. $2 B$ ). An AND ROI was placed on an axial slice capturing the UF bundle at the point where the bundle bends into the inferior temporal lobe region. This bend was visually identified on the midline sagittal plane with the axial slice being placed at a level immediately dorsal to the upper pons. A NOT ROI was drawn across the coronal slice level with the front of the pons to remove tracts of the internal frontal-occipital fasciculus. The tract was then visually inspected and any obvious outlier streamlines that were not consistent with the known UF anatomy (Crosby et al., 1962; Schmahmann and Pandya, 2006) were removed using additional NOT ROIs. This procedure was performed for both hemispheres.

Parahippocampal cingulum. A seed point ROI was placed on an axial slice level with the pons-midbrain junction around the cingulum fiber bundle that runs caudal and lateral to the pons (Fig. 2C). A NOT ROI was drawn across the midline sagittal plane to remove interhemispheric projections. After visual inspection, further NOT ROIs were placed, when necessary, to remove projections to the occipital lobe and any outlier tracts that were not consistent with the known anatomy of the PHC. This procedure was performed separately for the left and the right PHC.

Hippocampal volumes. The hippocampal formation is very closely associated with two of the tracts under investigation (the fornix and parahippocampal cingulum), as it has both efferent and afferent connections that comprise these tracts (Poletti and Creswell, 1977; Mufson and Pandya, 1984). This association is especially strong in the case of the fornix, where it is estimated that in the rhesus monkey $>95 \%$ of the temporal lobe cells projecting through the fornix originate in the hippocampus proper or the subicular cortices (Saunders and Aggleton, 2007). For these reasons, hippocampal volume was estimated to test for associations between the status of this structure and the status of the tracts under investigation. The hippocampus was singled out as it represents a special case-no other single site has such a strong affinity with any of the three tracts.

Hippocampal volumes were generated with HippoQuant software (Belaroussi et al., 2008) developed by BioClinica SAS. This is an adaptation of hippocampal outlining techniques described previously (Pruessner et al., 2000) but involves placing $\sim 80$ landmark points, rather than tracing full outlines on each slice, with the remaining surface filled in by deforming a standard three-dimensional hippocampal template to match these landmark points (Shen et al., 2002). First, T1-weighted images were displayed simultaneously in all three planes (axial, sagittal, coronal) and a trained rater placed multiple landmarks on the boundary of the hippocampus, paying particular attention to areas of difficult anatomical distinction (for example, the alveus and the boundary with the amygdala). The hippocampal measurements included the hippocampus proper (CA-1 to CA-4 sectors), dentate gyrus, alveus, fimbria, and subiculum. Parahippocampal regions (entorhinal cortex and intervening white matter including perforant pathway) were excluded. The resulting hippocampal mask was further refined by a tissue-type segmentation step (i.e., voxels segmented as gray or white matter are included, CSF voxels excluded). The final hippocampal masks were then checked visually by the rater and edited for obvious errors. The HippoQuant technique was validated in $30 \mathrm{MR}$ datasets extracted from an internal database representing various CNS diseases (MS, AD, stroke) by comparison with gold-standard manual delineation (Pruessner et al., $2000)$. This revealed a mean overlap of $>92 \%$ for voxels included in HippoQuant and gold-standard hippocampal masks (Belaroussi et al., 2008). Hippocampal volumes were corrected for head size by
Table 2. Correlation coefficients between the four episodic memory components identified by principal component analysis and age and fractional anisotropy of frontotemporal tracts

\begin{tabular}{|c|c|c|c|c|c|}
\hline & \multirow[b]{2}{*}{ Age } & \multirow[b]{2}{*}{ Fornix } & \multirow{2}{*}{$\begin{array}{l}\text { Uncinate } \\
\text { collapsed }\end{array}$} & \multicolumn{2}{|c|}{$\begin{array}{l}\text { Parahippocampal } \\
\text { cingulum }\end{array}$} \\
\hline & & & & Left & Right \\
\hline Age & - & $-0.65^{* * *}$ & $-0.49^{* *}$ & $-0.42^{* *}$ & -0.17 \\
\hline \multicolumn{6}{|l|}{ Memory components } \\
\hline General memory & $-0.44^{* *}$ & $0.48^{* *}$ & 0.01 & 0.13 & -0.03 \\
\hline Strategic memory & $-0.38^{*}$ & $0.40^{* *}$ & -0.17 & 0.27 & -0.06 \\
\hline Visual memory & $-0.45^{* *}$ & $0.48^{* *}$ & 0.23 & 0.19 & 0.01 \\
\hline Associative memory & $-0.48^{* *}$ & $0.50^{* * *}$ & -0.10 & 0.28 & 0.13 \\
\hline
\end{tabular}

calculating intracranial volume from summing segmented gray and white matter and CSF images (from the volumetric T1-weighted image) in Statistical Parametric Mapping 8 (Ashburner and Friston, 2005). Brain parenchymal fraction, i.e., the volume of gray and white matter divided by intracranial volume, as a measure for whole-brain volume was also obtained.

Statistical analyses. All statistical analyses were performed using SPSS 16.0 (SPSS). Since age effects were the focus and an effect of interest of this study, statistical analyses of cognitive performance were based on raw rather than age-scaled scores. The 13 episodic memory scores (Table 1) were of primary interest in the analyses, but a data reduction approach was also used to account for possible colinearities between these measures.

With principal component analysis, the following four memory components, explaining $76 \%$ of the variation in the data, were extracted: (1) general memory - the average of all memory measures except the PAL, (2) strategic memory-measures of encoding (FCSRT immediate recall) and cued recall (FCSRT total recall) as well as the PAL error ratio, (3) visual memory - the average of all visual memory tests in the Doors and People test and PAL, and (4) associative memory-the average of tests requiring some form of association; i.e., the PAL, verbal recognition of name pairs in the Doors and People test, and FCSRT cued recall. The terms for the components were purely descriptive of the type of the tasks they were derived from and were coined specifically for this study. Table 2 summarizes the correlation coefficients for these components with age and white matter microstructure.

Correlations with individual memory measures were controlled for multiple comparisons with the Bonferroni method, according to which individual comparisons had to reach a significance level of $p \leq 0.004$ to comply with an experiment-wise corrected significance level of 0.05 . Table 3 shows the correlation coefficients for each individual memory tests, including those not reaching experiment-wise significance.

Correlations between memory measures and white matter microstructure were controlled for potential confounds due to age, sex, and education. To further investigate whether variation in the episodic memory measures could be explained solely by age or reflected additional age-uncorrelated contributions from tract microstructure, hierarchical linear regression analyses were conducted based on individual memory measures. As there were only two white matter measures but 13 memory measures, initial exploratory analyses were performed using FA and MD as dependent variables. Age was entered as the first independent variable (Block 1) and all episodic memory scores in a stepwise fashion (Block 2) to the regression model. For memory measures found to have a significant association in this initial analysis, multiple regressions were repeated, this time using the episodic memory measure as the dependent variable. Finally, age and white matter correlations with measures of intelligence and executive functioning were explored (Table 1).

\section{Results}

Correlations between aging and memory components and individual memory measures

As can be seen from Table 1, participants, on average, performed at superior levels in measures of intelligence, performed well in 
Table 3. Correlation coefficients between individual episodic memory measures and age and fractional anisotropy of frontotemporal tracts

\begin{tabular}{|c|c|c|c|c|c|}
\hline & \multirow[b]{2}{*}{ Age } & \multirow[b]{2}{*}{ Fornix } & \multirow{2}{*}{$\begin{array}{l}\text { Uncinate } \\
\text { collapsed }\end{array}$} & \multicolumn{2}{|c|}{$\begin{array}{l}\text { Parahippocampal } \\
\text { cingulum }\end{array}$} \\
\hline & & & & Left & Right \\
\hline \multicolumn{6}{|l|}{ FCSRT } \\
\hline Immediate recall & $-0.52 * *$ & 0.30 & 0.19 & 0.05 & -0.10 \\
\hline Free recall & $-0.55^{* * *}$ & $0.57^{* * *}$ & 0.08 & 0.16 & 0.04 \\
\hline Total recall & $-0.47^{* *}$ & $0.49 * *$ & -0.17 & 0.30 & 0.02 \\
\hline Delayed free recall & $-0.48^{* *}$ & $0.43^{* *}$ & 0.08 & 0.19 & 0.07 \\
\hline Delayed total recall & $-0.46^{* *}$ & $0.43^{* *}$ & -0.53 & $0.36^{*}$ & 0.07 \\
\hline \multicolumn{6}{|l|}{ Doors and People } \\
\hline Visual recall & $-0.40^{* *}$ & $0.48 * *$ & 0.14 & 0.12 & -0.05 \\
\hline Verbal recall & -0.17 & 0.12 & 0.21 & -0.11 & -0.03 \\
\hline Delayed visual recall & $-0.33^{*}$ & $0.32^{*}$ & 0.01 & 0.25 & 0.07 \\
\hline Delayed verbal recall & -0.14 & 0.22 & 0.06 & 0.04 & 0.17 \\
\hline Visual recognition & $-0.36^{*}$ & $0.32^{*}$ & 0.25 & 0.18 & 0.04 \\
\hline Verbal recognition & $-0.37^{*}$ & $0.39^{*}$ & -0.08 & 0.16 & -0.01 \\
\hline \multicolumn{6}{|l|}{ Paired Associate Learning } \\
\hline Learning level & 0.27 & 0.25 & 0.25 & 0.12 & 0.05 \\
\hline Error ratio & 0.18 & -0.13 & $-0.49 * *$ & 0.28 & 0.11 \\
\hline
\end{tabular}

Correlations highlighted in bold were significant at $p \leq 0.004$ to comply with a Bonferonni-corrected experimentwise level of $0.05 .{ }^{*} p<0.05 ;{ }^{* *} p<0.01 ; * * * 0.001$.

the executive tasks, and showed no signs of depression. Participants also performed well in the episodic memory tasks and showed ceiling effects for some measures (Table 1). Ceiling effects in the FCSRT immediate recall measure illustrate that participants successfully encoded the learning material initially. Similarly, ceiling effects in the delayed recall measures demonstrate that after having been exposed to three immediate recall trials in the FCSRT and the Doors and People test, the majority of participants retained the material almost perfectly over a distraction period.

There were significant negative correlations between age and all four episodic memory components (Table 2). Individual analyses revealed significant negative correlations between age and all measures of recall in the FCSRT (Table 3). None of the other individual memory indices correlated with age after Bonferroni correction, although trends for negative correlations were observed in the Doors and People test.

\section{Correlations between age and white matter measures}

In the following description, FA and MD refer exclusively to partial volume-corrected measures. Age $(n=39)$ and fornix FA were significantly inversely correlated (Fig. 3 , Table 2 ), but there was no correlation between age and fornix $\operatorname{MD}(r=0.122, p=0.5)$.

For the UF, there were no significant differences between the slopes and intercepts of the linear regression fits between age and FA or MD between the left and right hemispheres. Hence, FA and MD measures for the UF were averaged across the hemispheres. There was a significant negative correlation between age and FA (Table 2) and a significant positive correlation between age and $\mathrm{MD}(r=0.622, p \leq 0.001)$.

For the PHC, there was a significant positive correlation between age and $\mathrm{MD}(r=0.54, p \leq 0.001)$ on the left but not on the right side $(r=0.19, p=0.18)$, with accompanying trends for FA (Table 2). Linear regression between age and FA revealed a significant difference between the slopes in left and right hemispheres $\left(t_{(74)}=2.2, p \leq 0.05\right)$, with a steeper and negative slope for the left side. Hence FA for PHC was not collapsed across hemispheres.

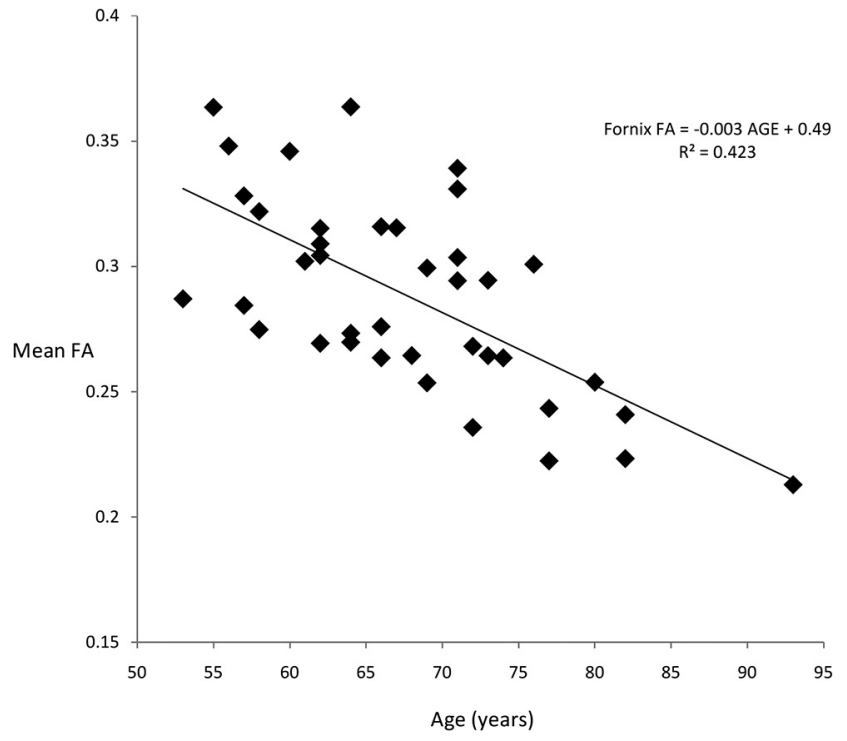

Figure 3. Relationship between age and fornix FA.

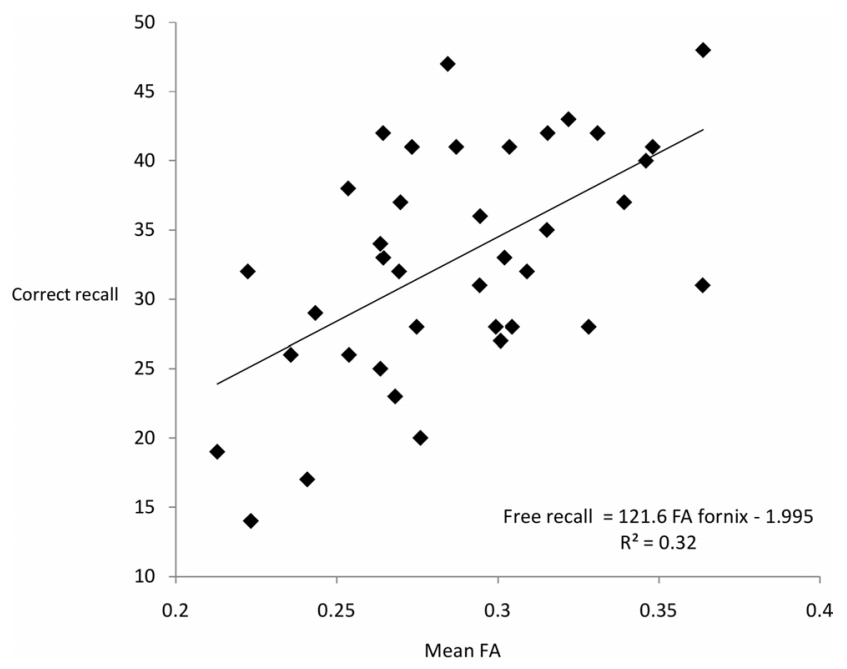

Figure 4. Relationship between fornix FA and immediate free recall in the free and cued selective reminding test.

\section{Correlations between white matter measures and episodic memory}

Fornix FA correlated significantly with all four memory components (Table 2) but no correlations were found between memory components and FA in the uncinate fasciculus or in the PHC. No correlations between memory components and MD in any of the white matter tracts were observed.

In the individual analyses, fornix FA correlated significantly with immediate free recall and total recall measures in the FCSRT (Fig. 4, Table 3) and there were correlations with delayed free recall and delayed total recall not reaching experiment-wise significance (Table 3). No correlation was found between FA and the FCSRT encoding measure (immediate recall). In addition, there was a significant positive correlation between fornix FA and immediate visual recall in the Doors and People test and trends toward association between fornix FA and delayed visual recall as well as visual and verbal recognition (Table 3). No correlations with verbal recall in the Doors and People test were observed. Ceiling effects were apparent in delayed measures, probably accounting for the discrepancy between the strengths in correla- 


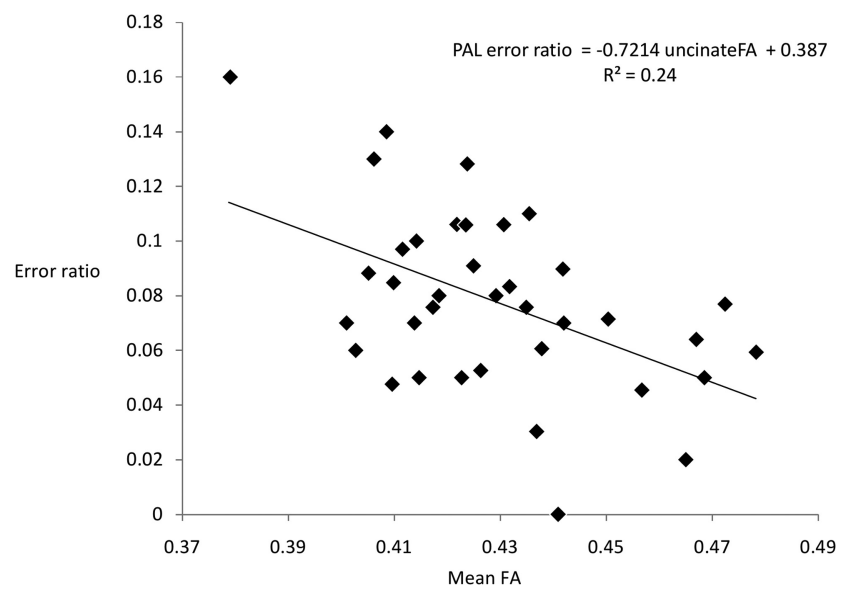

Figure 5. Relationship between average $F A$ in the uncinate fasciculi and the error ratio in the object-location paired associate task.

tions with immediate versus delayed measures (see also Vann et al., 2008). The same issue may account for the absence of a correlation with immediate recall in the FCSRT. Finally, no significant correlations were found between episodic memory measures and fornix MD.

For the uncinate fasciculus, a significant negative correlation between the error ratio in the Paired-Associate Learning task and FA averaged across hemispheres was found (Fig. 5, Table 3). No other significant correlations between FA or MD in the UF and memory were observed.

No significant correlations between microstructure in the $\mathrm{PHC}$ and episodic memory were found after Bonferonni correction. The strongest trends for association were between FA in the left PHC and delayed total recall in the FCSRT (Table 3), and MD in the left PHC and both total recall $(r=-0.38, p \leq 0.02)$ and total delayed recall $(r=-0.37, p \leq 0.02)$ in the FCSRT.

\section{Multivariate analysis and age-independent associations of white matter microstructure}

To investigate whether the observed associations between fornix FA and memory could be explained by age or other potentially relevant variables such as sex and education, partial correlations controlling for these confounds were carried out. When age was controlled for in the correlations between fornix FA and the four memory components, none of the correlations remained significant $(r=0.29, p=0.07$ for general memory; $r=0.23, p=0.18$ for strategic memory; $r=0.29, p=0.08$ for visual memory; $r=$ $0.3, p=0.07$ for association). Similarly, when age was controlled for in the correlations between fornix FA and individual memory measures, the correlations were not significant after Bonferonni correction ( $r=0.33, p=0.046$ for FCSRT free recall; $r=0.33$, $p=0.046$; and for visual recall; $r=0.26, p=0.10$ for FCSRT total recall).

To explore the relationship between age, fornix microstructure, and episodic memory beyond simple partial correlations, hierarchical regression analyses were carried out. In multivariate models, age alone accounted for $36 \%$ of the variation in fornix FA. The inclusion of visual recall, but no other memory measure, significantly improved the fit of the model. Regression analyses were therefore repeated with visual recall as the dependent variable, entering fornix FA and then age as independent variables. Fornix FA contributed significantly to the model and explained $23 \%$ of the variation in the visual recall performance. The inclusion of age did not significantly improve the fit of the model:
Visual recall $=26.3+0.38$ fornix FA $(t=2.1, p \leq 0.05)-0.17$ age $(t=-0.9, p=0.339)+$ error. Consistent results were observed when age was entered as the first and fornix FA as the second independent variable. This pattern of regression results demonstrates an association between fornix FA and visual recall that is uncorrelated with age and explains almost a quarter of the variance in visual recall performance.

Controlling for sex and education did not alter the pattern of results between fornix FA and memory components (general memory: $r=0.49, p \leq .002$ controlled for sex, $r=0.47, p \leq .003$ controlled for education; strategic memory: $r=0.40, p \leq 0.01$ controlled for sex, $r=0.39, p \leq 0.02$ controlled for education; visual memory: $r=0.47, p \leq .003$ controlled for sex, $r=0.47, p \leq$ .003 controlled for education; associative memory: $r=0.50, p \leq$ .002 controlled for sex, $r=0.50, p \leq .003$ controlled for education) or individual memory measures (FCSRT free recall: $r=0.53, p \leq$ .001 controlled for sex, $r=0.52, p \leq .001$ controlled for education; FCSRT total recall: $r=0.42, p \leq .009$ controlled for sex, $r=0.42, p \leq$ 0.01 controlled for education; visual recall: $r=0.48, p \leq .003$ controlled for sex, $r=0.484, p \leq .002$ controlled for education).

Finally, controlling for age, sex, and education did not change the significant correlation between FA in the uncinate fasciculus and the error ratio in the PAL $(r=-0.47, p \leq .004$ controlled for age, $r=-0.48, p \leq .003$ controlled for sex, $r=-0.45, p \leq .006$ controlled for education).

\section{Correlations between age, fornix white matter measures, and hippocampal volume}

There were no significant correlations between left and right hippocampal volume and fornix microstructure (right hippocampal volume and FA: $r=0.3, p=0.07$; right hippocampal volume and MD: $r=-0.115, p=0.496$; left hippocampal volume and FA: $r=0.254, p=0.129$; left hippocampal volume and MD: $r=$ $-0.054, p=0.752$ ).

A significant association was found between age and hippocampal volume on the right side $(r=-0.46, p \leq .004)$ but not on the left $(r=-0.38, p=0.02)$. However, when hippocampal volume was controlled for in the correlation between fornix FA and age, the correlation remained significant $(r=-0.588, p \leq$ $.001)$. Furthermore, partialling out hippocampal volume from the correlations between fornix FA and episodic memory did not affect the positive correlation with FCSRT free recall $(r=0.476$, $p \leq .004)$ and a trend remained with visual recall in the Doors and People test $(r=0.447, p \leq .007)$. Partialling out hippocampal volume from the correlations between fornix FA and the four memory components did not alter the pattern of results (general memory: $r=0.43, p \leq .009$; strategic memory: $r=0.35, p \leq 0.04$; visual memory: $r=0.45, p \leq .007$; associative memory: $r=0.47$, $p \leq .004)$.

\section{Correlations between white matter microstructure and age/ episodic memory controlled for whole-brain volume} Controlling for whole-brain atrophy with brain parenchymal fraction had no effect on the correlation between age and fornix FA $(r=-0.59, p \leq .001)$ but did attenuate correlations with the UF and PHC (UF: $r=-0.029, p=0.09$; PHC: $r=-0.087, p=$ $0.63)$. Controlling for brain volume did not change the main correlations between white matter microstructure and episodic memory (fornix FA-FCSRT free recall, $r=0.57, p \leq .001$; fornix FA-FCSRT total recall: $r=0.45, p \leq .008$; fornix FA-DP visual recall: $r=0.48, p \leq .004$; fornix FA-general memory: $r=0.48$, $p \leq .003$; strategic memory, $r=41, p \leq 0.01$; visual memory: $r=$ 
$0.46, p \leq .005$; associative memory, $r=-0.53, p \leq .005$; UF-PAL error ratio: $r=-0.47, p \leq .006$ ).

\section{Correlations with intelligence and executive functions}

No correlations between age and intelligence or executive functioning reached experiment-wise significance. However, trends were found for associations between age and category fluency $(r=-0.39, p \leq 0.01)$ and Digit Symbol $(r=-0.33, p \leq 0.05)$.

Similarly, no correlations between diffusion measures and intelligence/executive functioning reached experiment-wise significance. The strongest trends for association were between FA in the UF and category fluency $(r=0.34, p \leq 0.04)$, $\mathrm{MD}$ in the UF and visual span $(r=-0.33, p \leq 0.04)$, FA in the right PHC and Digit span backwards $(r=0.35, p \leq 0.03)$ and MD in the left PHC and errors in the verbal trails switching condition $(r=0.35, p \leq 0.03)$.

\section{Discussion}

Age-related variation in episodic memory in individuals aged over 50 was found to correlate specifically with microstructure of the fornix, a critical connection between MTL and both prefrontal and subcortical regions implicated in memory. In addition, performance in a visual object-location paired associate task correlated with microstructure of the uncinate fasciculus, which connects the anterior temporal lobe with prefrontal cortex regions. Microstructure of these frontotemporal connections was associated specifically with episodic memory; relationships with executive function were weaker and did not reach experimentwise significance. One important advance over previous studies (Sexton et al., 2010; Jang et al., 2011) is that these findings disentangle intrinsic microstructural change from the effects of tract atrophy.

Aging effects on partial volume-corrected fractional anisotropy and/or mean diffusivity were observed in all three frontotemporal fasciculi. However, the present study is the first to demonstrate that the relationship with episodic memory was specific to fornix microstructure. This discovery builds on studies of humans (Hodges and Carpenter, 1991; McMackin et al., 1995; Aggleton et al., 2000) and of animals (Gaffan, 1994; Aggleton and Brown, 2002) where complete fornix severance is associated with a loss of episodic-like memory. Multivariate analysis of the association between memory and fornix structure revealed that degradation in fornix microstructure was both associated with age-related and with age-uncorrelated changes in recall. These findings in a normal population strengthen the possibility that changes in this tract, alongside changes to the hippocampus itself, contribute to the memory loss seen in conditions such as schizophrenia (Kuroki et al., 2006; Fitzsimmons et al., 2009). Although in the present study, hippocampal volume also declined with age, covarying for hippocampal volume had little effect on this association. These results add substantially to our knowledge of cognitive aging by showing that the role of white matter microstructure extends beyond providing a general infrastructure for a wide range of attentional and other cognitive processes, to a more specific role for some tracts, for example in aspects of episodic memory.

Mean fornix fractional anisotropy values were positively associated with all four memory components, but individual test analyses demonstrated a specific association with performance in verbal and visual recall tasks. These results are consistent with the view that the fornix, together with the hippocampus, is critically and specifically involved in particular aspects of episodic memory (Aggleton et al., 2000; Rudebeck et al., 2009). This model posits distinct, but interleaved, networks for recollection and familiarity-based recognition (Aggleton and Brown, 2006), in which the fornix is vital for recollection. This model also emphasizes the importance of the hippocampal interactions with the mammillary bodies and thalamus, also via the fornix, which provide an alternate, indirect route to influence prefrontal cortex (Aggleton and Brown, 2006). The pattern of correlations between fornix FA and the various recall tasks is consistent with such a model. The one exception was verbal recall on the Doors and People task. Clinical studies show that there are hemispheric functional differences in the fornix that reflect the verbal-nonverbal distinction (Tucker et al., 1988), pointing to the need for even more fine grained analyses of tract status. In addition, the verbal recall tasks in Doors and People produce much greater variance than the equivalent nonvisual tasks (I. Nimmo-Smith, personal correspondence) (Table 1). Differences were also seen in the strengths of the correlations between fornix microstructure and recall versus recognition memory, thereby extending the findings from both neuropsychological (Aggleton et al., 2000) and imaging (Tsivilis et al., 2008) studies in patients with fornix damage. Dualprocess models assume that recognition memory relies both on recollection and familiarity-based processes (Yonelinas, 2002) and so one would expect this relatively weaker correlation with fornix microstructure for recognition than for recall.

Age effects were also observed in the uncinate fasciculus and parahippocampal cingulum but were attenuated after controlling for whole-brain volume. For the PHC, there was evidence of a significant asymmetry in aging effects, with the left side more susceptible to aging. The latter result adds to emerging evidence of asymmetries in white matter pathways and aging effects (Huster et al., 2009; Takao et al., 2010). Previous studies with diffusion MRI have also shown that aging effects are not uniform and vary in different white matter fasciculi (Jones et al., 2006).

The relationship between variations in white matter microstructure and age-related changes in item recall was specific to the fornix. However, for the UF, a significant and robust inverse relationship, uncorrelated with age, sex, or education, was found between the error ratio in paired-associate learning and fractional anisotropy: participants with the lowest fractional anisotropy values showed the highest error ratios and vice versa. In paired-associate learning, participants learned the association between a visual object and a spatial location and had to allocate each object to its correct location. Surgical disconnections in macaque monkeys have shown that bilateral UF lesions impair paired-association learning, specifically when a visual object cue has to be linked with a separate response choice (Eacott and Gaffan, 1992; Gaffan and Eacott, 1995a,b; Gutnikov et al., 1997). The same lesions, however, had no effect on item memory (Gaffan and Eacott, 1995a), configural learning (Gutnikov et al., 1997), or associative learning involving nonvisual cues (Eacott and Gaffan, 1992). Our finding of significant associations between UF microstructure and the error ratio in the visual pairedassociate learning task is consistent with these results and provides the first evidence for a similar functional role of the UF in humans. Together, they suggest that the UF may be critically involved in visual associative learning in contrast to item memory supported by MTL structures. As the UF projects to the orbitofrontal cortex, proposed to support error-related monitoring processes in interaction with the anterior cingulate and lateral prefrontal cortex (Turken and Swick, 2008), one might further speculate that the UF mediates feedback mechanisms involved in the monitoring and evaluation of ongoing responses to visual object cues. 
No correlations between episodic memory measures and the $\mathrm{PHC}$ were found, reinforcing the view that the fornix is the more critical connection with the MTL. However, there were some trends in the data linking PHC microstructure to total recall in the FCSRT. Recent evidence suggests that the posteromedial cortex extending into the posterior cingulate cortex is critical for strategic memory processes related to successful encoding/retrieval and meta-memory (Daselaar et al., 2009). Thus, it may be the case that the PHC contributes to strategic aspects of memory not directly assessed in the present study.

Although both were correlated with age, no correlation was found between hippocampal volume and fornix microstructure. The fornix is a large, complex fiber tract, containing efferent projections from both the hippocampus and parahippocampal region, as well as afferent connections from subcortical and brainstem nuclei (Saunders and Aggleton, 2007). One interpretation of the present data is that aging has a direct effect on connections via changes in white matter structure. This account would be consistent with those neuropathological studies suggesting a selective loss of small, myelinated fibers but no substantial neuronal loss in aging (Morrison and Hof, 1997; Tang et al., 1997). Alternatively, it could be argued that degeneration of some subset of neurons in aging has a relatively greater effect on fornix microstructure than on hippocampal volume.

Aging is associated with gray and white matter tissue loss, and the resulting partial volume errors can lead to artifactual decreases in fractional anisotropy and increases in mean diffusivity due to CSF contamination. Recent evidence indicates that diffusivity measures compared with FA may be more prone to CSFbased partial volume artifacts (Pasternak et al., 2009; Vos et al., 2011) and hence may not provide reliable measures of white matter microstructure in the presence of significant atrophy. Thus, in the present study, we reported only partial volume corrected measures of fractional anisotropy and mean diffusivity, and this is the likely reason for the lack of a significant correlation between mean diffusivity measures in the fornix and age, in contrast to previous studies that did not control for age- or pathologyrelated volume loss (Sexton et al., 2010; Jang et al., 2011).

The findings of the present study have to be interpreted within the limitations of a cross-sectional design, which is susceptible to cohort effects and reflects only chronological age. Inferences about the causality of the reported effects are also difficult based on correlation alone. Our study focused on three tracts thought to be important for episodic memory based on animal studies. Naturally, there may be other tracts, for instance, within the thalamofrontal network, that may be relevant to memory and susceptible to age and hence contributing to aging effects.

In summary, variation in fornix microstructure mediates agerelated decline but also age-uncorrelated variation in episodic memory. The pattern of associations with cognitive performance, for both fornix and uncinate fasciculus, correspond to predictions from experimental disconnections in rodents and monkeys, and thereby add considerable support to models describing these interactions. Individual differences in white matter microstructure could be a key factor in explaining interindividual variations in cognitive aging.

\section{References}

Aggleton JP, Brown MW (2002) Integrating systems for event memory: Testing the contribution of the fornix. In: Neuropsychology of memory, third edition (Squire LR, Schacter D, eds), pp. 377-394. New York: Guilford Publications.

Aggleton JP, Brown MW (2006) Interleaving brain systems for episodic and recognition memory. Trends Cogn Sci 10:455-463.
Aggleton JP, McMackin D, Carpenter K, Hornak J, Kapur N, Halpin S, Wiles CM, Kamel H, Brennan P, Carton S, Gaffan D (2000) Differential cognitive effects of colloid cysts in the third ventricle that spare or compromise the fornix. Brain 123:800-815.

Ashburner J, Friston KJ (2005) Unified segmentation. Neuroimage 26:839-851.

Baddeley AD (1996) Exploring the central executive. Q J Exp Psych 49 A:5-28.

Baddeley AD, Emslie H, Nimmo-Smith I (1994) Doors and People test. Bury St Edmunds: Thames Valley Test Company.

Barrick TR, Charlton RA, Clark CA, Markus HS (2010) White matter structural decline in normal aging: a prospective longitudinal study using tract-based spatial statistics. Neuroimage 51:565-577.

Basser PJ, Mattiello J, LeBihan D (1994) Estimation of the effective selfdiffusion tensor from the NMR spin echo. J Magn Reson B 103: 247-254.

Basser PJ, Pajevic S, Pierpaoli C, Duda J, Aldroubi A (2000) In vivo fiber tractography using DT-MRI data. Magn Reson Med 44:625-632.

Belaroussi B, O’Sullivan M, Vincent F, Pachai C (2008) HippoQuant: combining geometrical and intensity information for 3D hippocampus detection in 3D T1-weighted MRI images. Paper presented at 16th Scientific Meeting and Exhibition of the International Society in Magnetic Resonance in Medicine, Toronto, May.

Blackwell AD, Sahakian BJ, Vesey R, Semple JM, Robbins TW, Hodges JR (2004) Detecting dementia: novel neuropsychological markers of preclinical Alzheimer's disease. Dement Geriatr Cogn Disord 17: $42-48$.

Cabeza R, Ciaramelli E, Olson IR, Moscovitch M (2008) The parietal cortex and episodic memory: an attentional account. Nat Rev Neurosci 9:613-625.

Catani M, Howard RJ, Pajevic S, Jones DK (2002) Virtual in vivo interactive dissection of white matter fasciculi in the human brain. Neuroimage 17:77-94.

Concha L, Gross DW, Beaulieu C (2005) Diffusion tensor tractography of the limbic system. AJNR Am J Neuroradiol 26:2267-2274.

Crosby EC, Humphrey T, Lauer EW (1962) Correlative anatomy of the nervous system. New York: Macmillan.

Daselaar SM, Prince SE, Dennis NA, Hayes SM, Kim H, Cabeza R (2009) Posterior midline and ventral parietal activity is associated with retrieval success and encoding failure. Front Hum Neurosci 3:13.

Delis DC, Kaplan E, Kramer JH (2001) Delis-Kaplan Executive Function System (D-KEFS). Oxford, UK: Pearson Assessment.

Della Sala S, Wilson L, Gray C (1997) Visual Pattern Test. Oxford, UK: Pearson Assessment.

Eacott MJ, Gaffan D (1992) Inferotemporal-frontal disconnection: the uncinate fascicle and visual associative learning in monkeys. Eur J Neurosci 4:1320-1332.

Fazekas F, Kleinert R, Offenbacher H, Schmidt R, Kleinert G, Payer F, Radner H, Lechner H (1993) Pathological correlates of incidental MRI white matter signal hyperintensities. Neurology 43:1683-1689.

Fitzsimmons J, Kubicki M, Smith K, Bushell G, Estepar RS, Westin CF, Nestor PG, Niznikiewicz MA, Kikinis R, McCarley RW, Shenton ME (2009) Diffusion tractography of the fornix in schizophrenia. Schizophr Res 107:39-46.

Fletcher PC, Henson RN (2001) Frontal lobes and human memory: insights from functional neuroimaging. Brain 124:849-881.

Gaffan D (1994) Scene-specific memory for objects: a model of episodic memory impairment in monkeys with fornix transection. J Cogn Neurosci 6:305-320.

Gaffan D, Eacott MJ (1995a) Uncinate fascicle section leaves delayed matching-to-sample intact, with both large and small stimulus sets. Exp Brain Res 105:175-180.

Gaffan D, Eacott MJ (1995b) Visual learning for an auditory secondary reinforcer by macaques is intact after uncinate fascicle section: indirect evidence for the involvement of the corpus striatum. Eur J Neurosci 7:1866-1871.

Gaffan D, Wilson CR (2008) Medial temporal and prefrontal function: recent behavioural disconnection studies in the macaque monkey. Cortex 44:928-935.

Ge Y, Grossman RI, Babb JS, Rabin ML, Mannon LJ, Kolson DL (2002) Age-related total gray matter and white matter changes in normal adult 
brain. Part I: volumetric MR imaging analysis. AJNR Am J Neuroradiol 23:1327-1333.

Grober E, Buschke H (1987) Genuine memory deficits in dementia. Dev Neuropsychol 3:13-36.

Gutnikov SA, Ma YY, Buckley MJ, Gaffan D (1997) Monkeys can associate visual stimuli with reward delayed by $1 \mathrm{~s}$ even after perirhinal cortex ablation, uncinate fascicle section or amygdalectomy. Behav Brain Res 87:85-96.

Hodges JR, Carpenter K (1991) Anterograde amnesia with fornix damage following removal of IIIrd ventricle colloid cyst. J Neurol Neurosurg Psychiat 54:633-638.

Howard D, Patterson K (1992) Pyramids and palm trees: a test of semantic access from pictures and words. Bury St. Edmunds, UK: Thames Valley Test Company.

Huster RJ, Westerhausen R, Kreuder F, Schweiger E, Wittling W (2009) Hemispheric and gender related differences in the midcingulum bundle: a DTI study. Hum Brain Mapp 30:383-391.

Jang SH, Cho SH, Chang MC (2011) Age-related degeneration of the fornix in the human brain: a diffusion tensor imaging study. Int J Neurosci 121:94-100.

Jones DK, Cercignani M (2010) Twenty-five pitfalls in the analysis of diffusion MRI data. NMR Biomed 23:803-820.

Jones DK, Simmons A, Williams SC, Horsfield MA (1999a) Non-invasive assessment of axonal fiber connectivity in the human brain via diffusion tensor MRI. Magn Reson Med 42:37-41.

Jones DK, Horsfield MA, Simmons A (1999b) Optimal strategies for measuring diffusion in anisotropic systems by magnetic resonance imaging. Magn Reson Med 42:515-525.

Jones DK, Travis AR, Eden G, Pierpaoli C, Basser PJ (2005) PASTA: pointwise assessment of streamline tractography attributes. Magn Reson Med 53:1462-1467.

Jones DK, Catani M, Pierpaoli C, Reeves SJ, Shergill SS, O’Sullivan M, Golesworthy P, McGuire P, Horsfield MA, Simmons A, Williams SC, Howard RJ (2006) Age effects on diffusion tensor magnetic resonance imaging tractography measures of frontal cortex connections in schizophrenia. Hum Brain Mapp 27:230-238.

Kuroki N, Kubicki M, Nestor PG, Salisbury DF, Park HJ, Levitt JJ, Woolston S, Frumin M, Niznikiewicz M, Westin CF, Maier SE, McCarley RW, Shenton ME (2006) Fornix integrity and hippocampal volume in male schizophrenic patients. Biol Psychiat 60:22-31.

Leemans A, Jones DK (2009) The B-matrix must be rotated when correcting for subject motion in DTI data. Magn Reson Med 61:1336-1349.

Leemans A, Jeurissen B, Siibers J, Jones DK (2009) ExploreDTI: a graphical tool box for processing, analyzing, and visualizing diffusion MR data. Paper presented at 17 th Anuual Meeting of the International Society of Magnetic Resonance in Medicine, Hawaii.

Levine B, Black SE, Cabeza R, Sinden M, Mcintosh AR, Toth JP, Tulving E, Stuss DT (1998) Episodic memory and the self in a case of isolated retrograde amnesia. Brain 121:1951-1973.

Mabbott DJ, Rovet J, Noseworthy MD, Smith ML, Rockel C (2009) The relations between white matter and declarative memory in older children and adolescents. Brain Res 1294:80-90.

Malykhin N, Concha L, Seres P, Beaulieu C, Coupland NJ (2008) Diffusion tensor imaging tractography and reliability analysis for limbic and paralimbic white matter tracts. Psychiatry Res 164:132-142.

McKenna P, Warrington EK (1980) Testing for nominal dysphasia. J Neurol Neurosurg Psychiatry 43:781-788.

McMackin D, Cockburn J, Anslow P, Gaffan D (1995) Correlation of fornix damage with memory impairment in six cases of colloid cyst removal. Acta Neurochir (Wien) 135:12-18.

Morrison JH, Hof PR (1997) Life and death of neurons in the aging brain. Science 278:412-419.

Mufson EJ, Pandya DN (1984) Some observations on the course and composition of the cingulum bundle in the rhesus monkey. J Comp Neurol 225:31-43.

Nelson HE (1991) The National Adult Reading Test-Revised (NART-R): test manual. Windsor, UK: National Foundation for Educational Research-Nelson.

O'Sullivan M, Jones DK, Summers PE, Morris RG, Williams SC, Markus HS (2001) Evidence for cortical "disconnection" as a mechanism of agerelated cognitive decline. Neurology 57:632-638.

Pajevic S, Pierpaoli C (1999) Color schemes to represent orientation of anisotropic tissues from diffusion tensor data: application to white matter fiber tract mapping in the human brain. Magn Reson Med 42:526-540.

Pasternak O, Sochen N, Gur Y, Intrator N, Assaf Y (2009) Free water elimination and mapping from diffusion MRI. Magn Reson Med 62:717-730.

Pierpaoli C, Jones DK (2004) Removing CSF contamination in brain DTMRIs by using a two-compartment tensor model. Presented at ISMRM 12th Annual Meeting, Kyoto.

Pierpaoli C, Barnett A, Pajevic S, Chen R, Penix LR, Virta A, Basser P (2001) Water diffusion changes in Wallerian degeneration and their dependence on white matter architecture. Neuroimage 13:1174-1185.

Poletti CE, Creswell G (1977) Fornix system efferent projections in the squirrel monkey: an experimental degeneration study. J Comp Neurol 175:101-128.

Pruessner JC, Li LM, Serles W, Pruessner M, Collins DL, Kabani N, Lupien S, Evans AC (2000) Volumetry of hippocampus and amygdala with highresolution MRI and three-dimensional analysis software: minimising the discrepancies between laboratories. Cereb Cortex 10:433-442.

Ranganath C (2010) A unified framework for the functional organization of the medial temporal lobes and the phenomenology of episodic memory. Hippocampus 20:1263-1290.

Rudebeck SR, Scholz J, Millington R, Rohenkohl G, Johansen-Berg H, Lee AC (2009) Fornix microstructure correlates with recollection but not familiarity memory. J Neurosci 29:14987-14992.

Sasson E, Doniger GM, Pasternak O, Assaf Y (2010) Structural correlates of memory performance with diffusion tensor imaging. Neuroimage 50:1231-1242.

Saunders RC, Aggleton JP (2007) Origin and topography of fibers contributing to the fornix in macaque monkeys. Hippocampus 17:396-411.

Schmahmann JD, Pandya DN (2006) Fiber pathways of the brain. New York: Oxford UP.

Sexton CE, Mackay CE, Lonie JA, Bastin ME, Terrière E, O'Carroll RE, Ebmeier KP (2010) MRI correlates of episodic memory in Alzheimer's disease, mild cognitive impairment, and healthy aging. Psychiatry Res 184:57-62.

Sheikh JI, Yesavage JA (1986) Geriatric Depression Scale (GDS): recent evidence and development of a shorter version. In: Clinical gerontology: a guide to assessment and intervention, pp 165-173. New York: Haworth.

Shen D, Moffat S, Resnick SM, Davatzikos C (2002) Measuring size and shape of the hippocampus in MR images using a deformable shape model. Neuroimage 15:422-434.

Simons JS, Spiers HJ (2003) Prefrontal and medial temporal lobe interactions in long-term memory. Nat Rev Neurosci 4:637-648.

Smith CD, Chebrolu H, Wekstein DR, Schmitt FA, Jicha GA, Cooper G, Markesbery WR (2007) Brain structural alterations before mild cognitive impairment. Neurology 68:1268-1273.

Stufflebeam SM, Witzel T, Mikulski S, Hämäläinen MS, Temereanca S, Barton JJ, Tuch DS, Manoach DS (2008) A non-invasive method to relate the timing of neural activity to white matter microstructural integrity. Neuroimage 42:710-716.

Takao H, Abe O, Yamasue H, Aoki S, Kasai K, Sasaki H, Ohtomo K (2010) Aging effects on cerebral asymmetry: a voxel-based morphometry and diffusion tensor imaging study. Magn Reson Imaging 28:65-69.

Tang Y, Nyengaard JR, Pakkenberg B, Gundersen HJ (1997) Age-induced white matter changes in the human brain: a stereological investigation. Neurobiol Aging 18:609-615.

Tournier JD, Calamante F, Gadian DG, Connelly A (2004) Direct estimation of the fiber orientation density function from diffusionweighted MRI data using spherical deconvolution. Neuroimage 23:1176-1185.

Tournier JD, Calamante F, Connelly A (2007) Robust determination of the fibre orientation distribution in diffusion MRI: non-negativity constrained super-resolved spherical deconvolution. Neuroimage 35:1459-1472.

Tournier JD, Yeh CH, Calamante F, Cho KH, Connelly A, Lin CP (2008) Resolving crossing fibres using constrained spherical deconvolution: validation using diffusion-weighted imaging phantom data. Neuroimage 42:617-625.

Trenerry MR, Crosson B, DeBoe J, Leber WR (1989) Stroop Neuropsychological Screening Test. Odessa, FL: Psychological Assessment Resources. 
Tsivilis D, Vann SD, Denby C, Roberts N, Mayes AR, Montaldi D, Aggleton JP (2008) A disproportionate role for the fornix and mammillary bodies in recall versus recognition memory. Nat Neurosci 11:834-842.

Tucker DM, Roeltgen DP, Tully R, Hartmann J, Boxell C (1988) Memory dysfunction following unilateral transection of the fornix: a hippocampal disconnection syndrome. Cortex 24:465-472.

Turken AU, Swick D (2008) The effect of orbitofrontal lesions on the errorrelated negativity. Neurosci Lett 441:7-10.

Valenstein E, Bowers D, Verfaellie M, Heilman KM, Day A, Watson RT (1987) Retrosplenial amnesia. Brain 110:1631-1646.

Vann SD, Denby C, Love S, Montaldi D, Renowden S, Coakham HB (2008) Memory loss resulting from fornix and septal damage: impaired supra- span recall but preserved recognition over a 24 -hour delay. Neuropsychology 22:658-668.

Vann SD, Aggleton JP, Maguire EA (2009) What does the retrosplenial cortex do? Nat Rev Neurosci 10:792-802.

Vos SB, Jones DK, Viergever MA, Leemans A (2011) Partial volume effect as a hidden covariate in DTI analyses. Neuroimage 55:1566-1576.

Wechsler D (1999) Wechsler Adult Intelligence Scale-3rd UK Edition (WAIS-III UK). Oxford, UK: Psychological Corporation and Pearson Assessment.

Wilding EL, Herron JE (2006) Electrophysiological measures of episodic memory control and memory retrieval. Clin EEG Neurosci 37:315-321.

Yonelinas AP (2002) The nature of recollection and familiarity: a review of 30 years of research. J Mem Lang 46:441-517. 